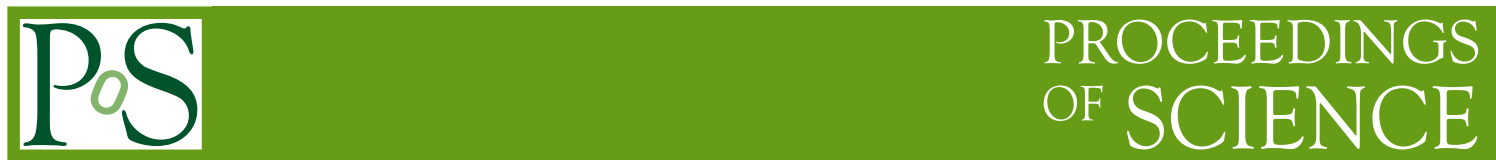

\title{
Multidimensional Hadron Attenuation
}

\section{Gevorg Karyan *i}

A. I. Alikhanyan National Science Laboratory, Yerevan, Armenia

E-mail: gevkar@mail.desy.de

Hadron multiplicity ratios in semi-inclusive deep-inelastic scattering have been measured on neon, krypton and xenon targets relative to deuterium using a $27.6 \mathrm{GeV}$ positron or electron beam at the HERMES experiment. They are presented for pions $\left(\pi^{+}, \pi^{-}\right), \operatorname{kaons}\left(K^{+}, K^{-}\right)$and protons $(p, \bar{p})$ as a function of the virtual photon energy $v$, its virtuality $Q^{2}$, the fractional hadron energy $z$ and the square of transverse hadron momentum $p_{t}^{2}$ with respect to the direction of the virtual photon. Dependencies are presented in a two-dimensional representation, in the form of detailed binning over one variable and three slices over the other variable. These results may help to understand some aspects of the hadronization process.

50th International Winter Meeting on Nuclear Physics - Bormio2012,

23-27 January 2012

Bormio, Italy

\footnotetext{
${ }^{*}$ Speaker.

$\dagger$ for the HERMES Collaboration
} 

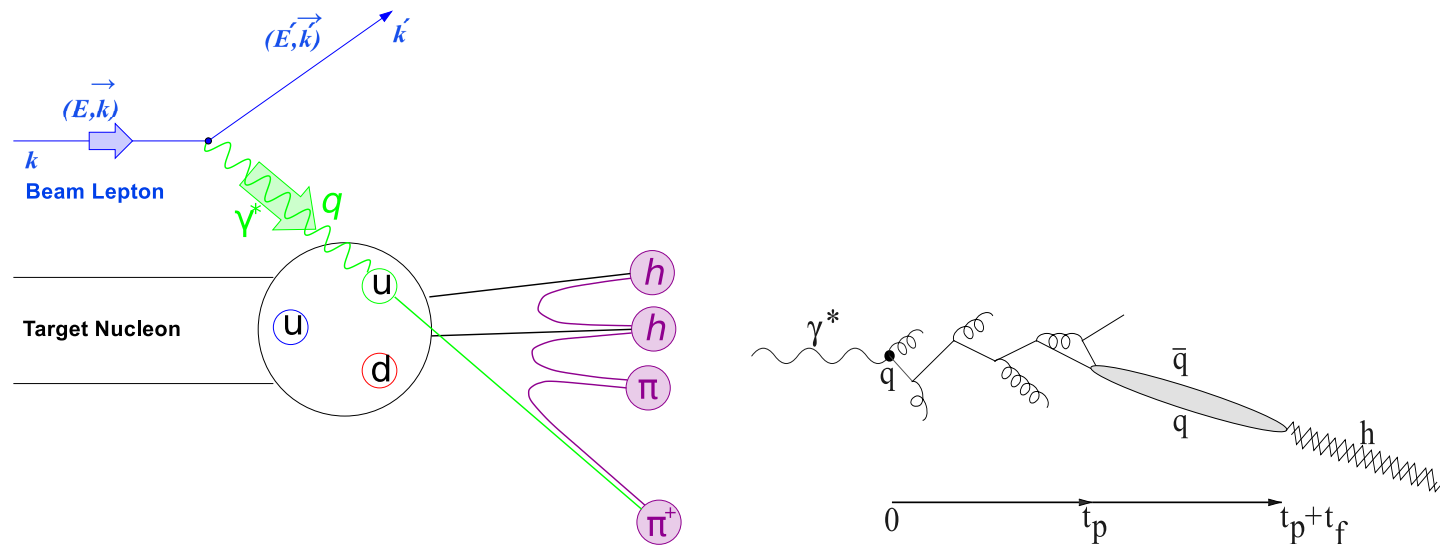

Figure 1: A simple illustration of the hadronization process( left diagram ) and it's space-time development( right diagram ) in semi-inclusive deep-inelastic scattering reaction.

\section{Introduction}

Semi-inclusive deep-inelastic scattering (SIDIS) of leptons from nuclei provides the tool to investigate a quark hadronization or fragmentation into hadrons[1]组. In such a process, lepton transfers a certain amount of energy $(v)$ to the "struck" quark which then propagates through the nuclear medium, losing it's energy by emiting a gluon. The time (length) is needed for this propagation is called the production time ( $t_{p}$, figure 1$)$. After this time a colorless pre-hadron is formed, which evolves to the final hadron during the formation time $\left(t_{f}\right.$, figure 1$)$. In order to investigate a space-time evolution of this process, nuclei with different mass (size) can be used. The experimental observable is the nuclear attenuation ratio, which is a ratio between hadron multiplicities from nuclear target with atomic mass (A) to those on the deuterium[3] [4] [5].

$$
R_{A}^{h}\left(v, Q^{2}, z, p_{t}^{2}\right)=\frac{\left(\frac{N^{h}\left(v, Q^{2}, z, p_{t}^{2}\right)}{N^{e}\left(v, Q^{2}\right)}\right)_{A}}{\left(\frac{N^{h}\left(v, Q^{2}, z, p_{t}^{2}\right)}{N^{e}\left(v, Q^{2}\right)}\right)_{D}}
$$

where $N^{h}$ is the number of semi-inclusive hadrons in a given $\left(v, Q^{2}, z, p_{t}^{2}\right)$ bin and $N^{e}$ is the number of inclusive deep-inelastic scattered leptons in the same $\left(v, Q^{2}\right)$ bin.

This ratio depends on two leptonic variables: the energy of virtual photon $v$ and it's virtuality $Q^{2}$ and on two hadronic variables: the fraction $z$ of the virtual-photon energy carried by the hadron and the square of the hadron momentum component $p_{t}^{2}$ transverse to the virtual-photon direction. The attenuation ratio, in general, depends also on azimuthal angle $\phi$, which is an angle between the lepton-scattering plane and the hadron-production plane. In this measurement no $\phi$ dependence was observed within statistical accuracy. Thus the integration over $\phi$ was performed.

The dependencies of $R_{A}^{h}$ were presented in a two-dimensional form, which means the detailed binning over one variable and and a coarser binning in another variable. The other variables are integrated over within the acceptance of the experiment. This allows to study the nuclear attenuation effect in more detail. 


\section{Data Extraction}

The data were collected with the HERMES spectrometer using $27.6 \mathrm{GeV}$ electron or positron beams stored in HERA at DESY[6]. To select deep-inelastic scattered (DIS) lepton the following

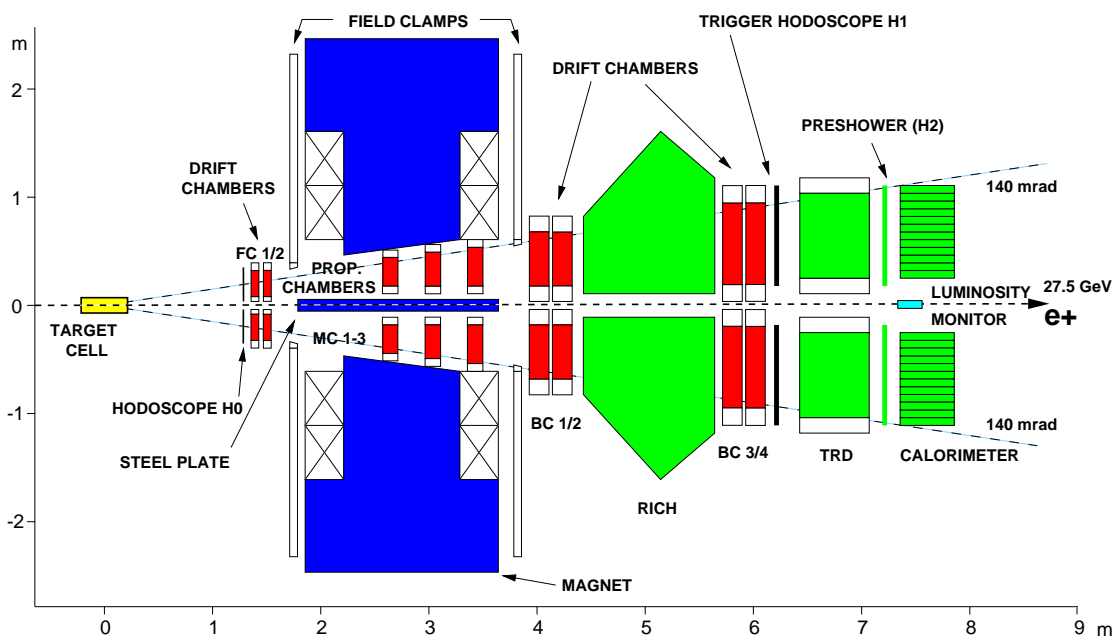

Figure 2: The HERMES spectrometer.

requirements were used for it's kinematics: $Q^{2}>1 \mathrm{GeV}^{2}, W^{2}>4 \mathrm{GeV}^{2}$ and $y=v / E<0.85$, where $W$ is the invariant mass of virtual photon-nucleon system and $E$ is the beam energy. The cut on the invariant mass imposed to suppress the resonance contribution into data and constrain on $y$ limits the magnitude of radiative corrections.

For charged hadron identification, dual-radiator ring-imaging Čerenkov detector (RICH) was used[7], which allows to identify pions, kaons and (anti)proton. They were selected within the following kinematical conditions: $2<p_{h}<15.0 \mathrm{GeV}, x_{F}>0$ and $z>0.2$, where $p_{h}$ is the hadron momentum

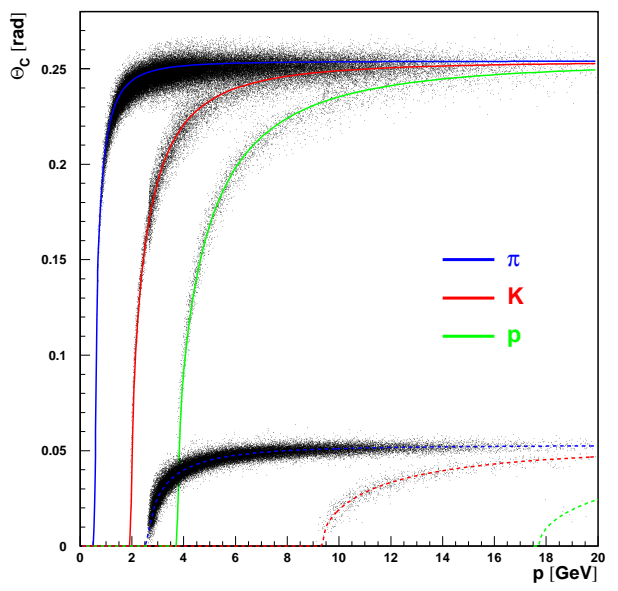

Figure 3: Momentum dependence of the Čerenkov angle for different hadron types and radiators. The upper band corresponds to the aerogel and the lower band to the $\mathrm{C}_{4} \mathrm{~F}_{10}$ gas respectively.

and $x_{F}$ is the Feynman variable which is defined as a ratio of the longitudinal momentum transferred 
to the hadron in the photon-nucleon centre-of-mass system to it's maximum possible value. In order to select hadrons coming from current fragmentation region the constraints on $x_{F}$ and $z$ applied, which reduce the contribution of target fragmentation into data.

\section{Results}

The results of $R_{A}^{h}$ are presented for neon (Ne), krypton (Kr) and xenon (Xe) for charge separated pions, kaons and protons using a fine binning in one of the kinematical variables and three slices in a second variable.
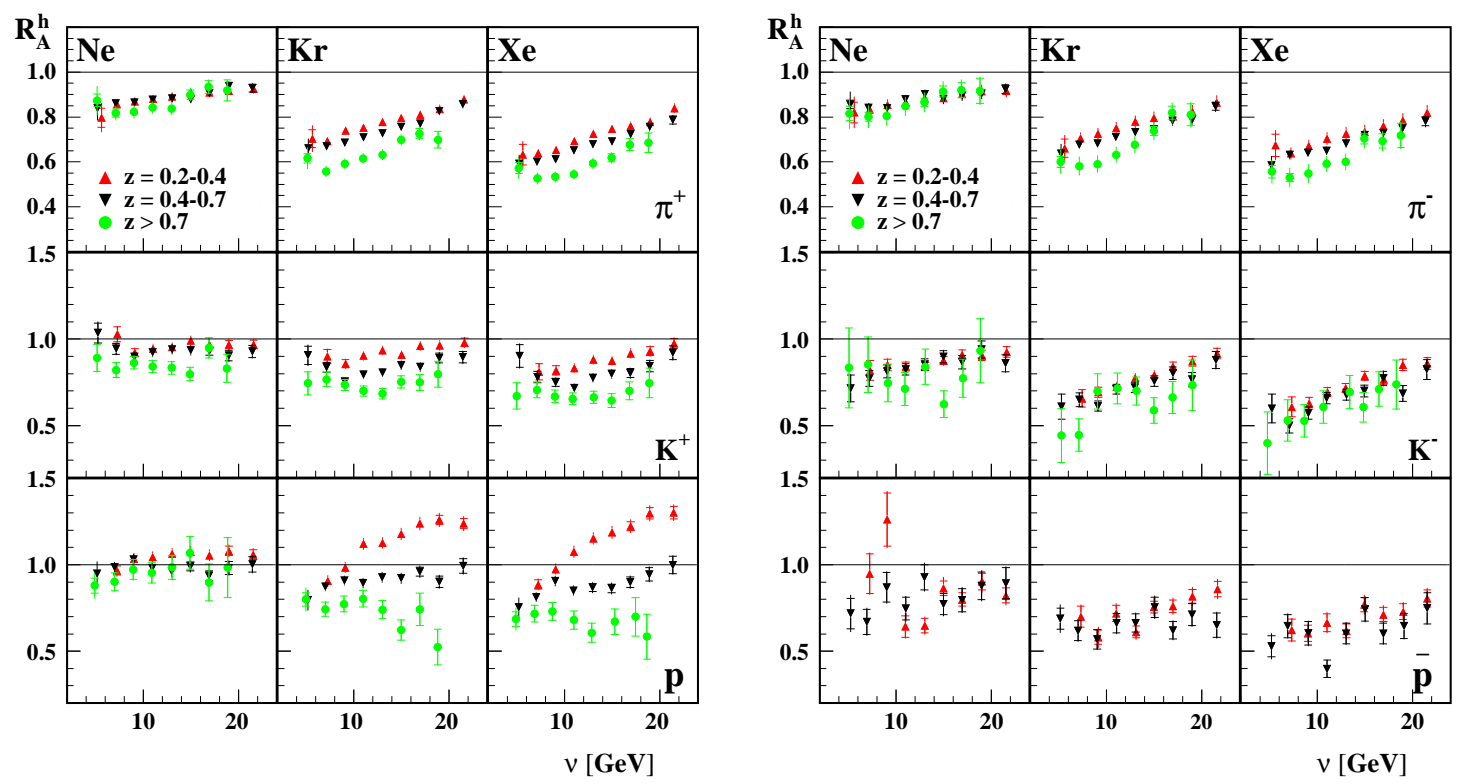

Figure 4: Dependence of $R_{A}^{h}$ on $v$ for three slices in $z$.

In figure 4, the $v$ dependence of $R_{A}^{h}$ is shown in three $z$ slices. The scale uncertainties are estimated to be $3 \%, 5 \%, 4 \%$, and $10 \%$ for pions, kaons, protons and atiprotons, respectively. With the increasing of $v$ the enhancement of $R_{A}^{h}$ was observed for pions $\left(\pi^{+}, \pi^{-}\right)$and negatively charged $\operatorname{kaons}\left(K^{-}\right)$ which is consistent with fragmentation models, explaining such a behaviour as a result of Lorentz dilation and/or a modification of the fragmentation function[8]. In contrast to negatively charged kaons $\left(K^{-}\right)$, the $v$ dependence of $R_{A}^{h}$ for positively charged kaons $\left(K^{+}\right)$shows an enhancement for the lowest $z$-slice and it seems flatter for the high $z$ values. For protons the behaviour of $R_{A}^{h}$ is very different from those for the other hadrons. Particularly for the lowest z-slice it exceeds the unity at large $v$ values. This phenomena might be caused by the fact that protons apart from hadronization could be knocked out off the nucleus while other hadrons are the result of hadronization only. This effect is stronger for heavy nuclei which is consistent with the assumption of large contribution from knock-out processes. Despite a lack of statistics, antiprotons, unlike protons, show a similar behaviour as mesons.

In figure 5, the dependence of $R_{A}^{h}$ on $z$ for three slices in $v$ is presented. The $R_{A}^{h}$ shows a slight change in different $v$ slices for $\pi^{+}$and $\pi^{-}$and a strong dependence for protons on krypton and xenon targets. This behaviour for protons can be explained by a large contribution from final-state 
interaction at low $z$ values.
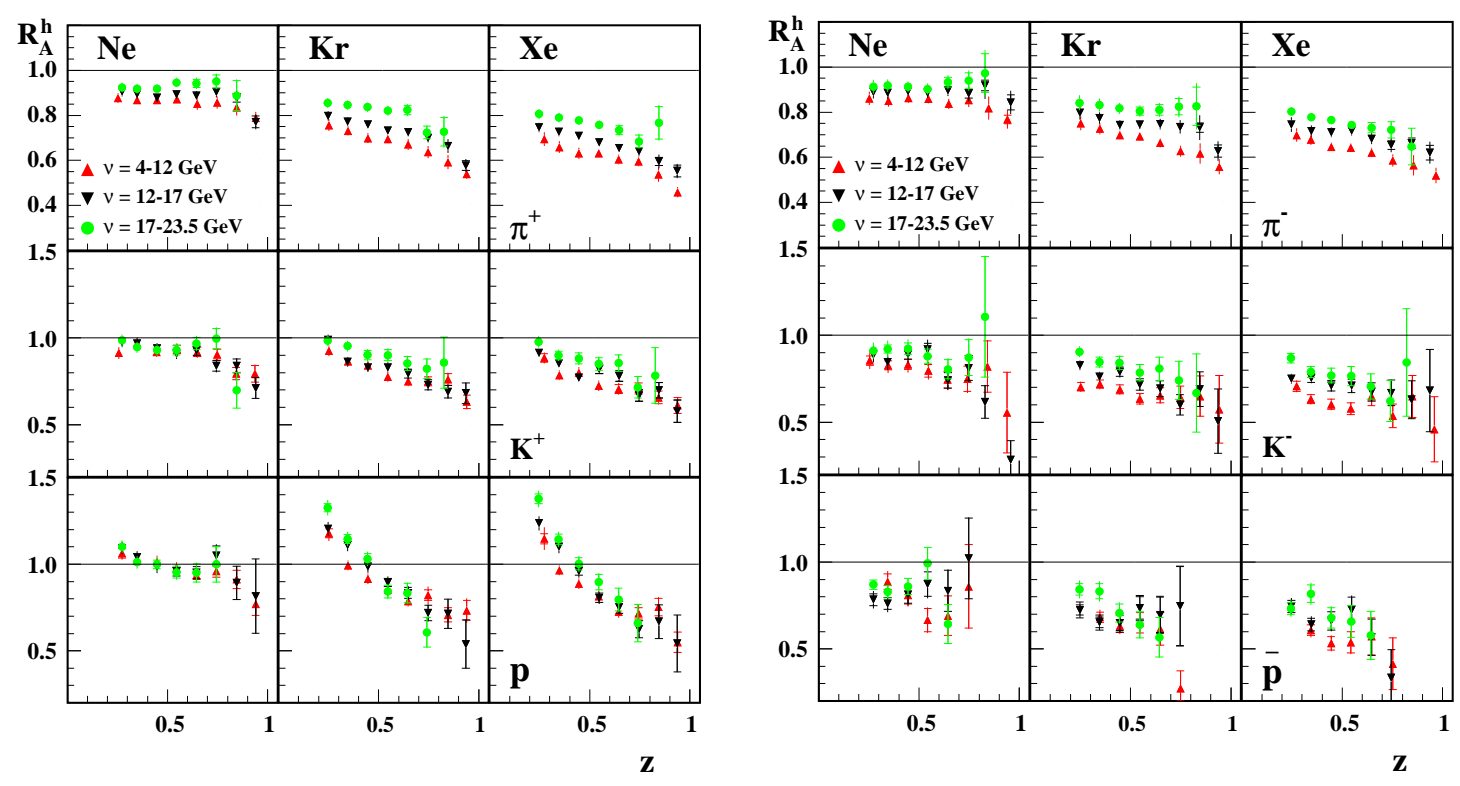

Figure 5: Dependence of $R_{A}^{h}$ on $z$ for three slices in $v$.

The dependence of $R_{A}^{h}$ on $p_{t}^{2}$ is shown in figure 6 for three slices in $z$. An increasing behaviour of $R_{A}^{h}$ was observed at high $p_{t}^{2}$ (the Cronin effect) which is larger for protons compared with mesons. For the highest $z$ slice the Cronin effect is suppressed for mesons while the protons show a significant rise with $p_{t}^{2}$.

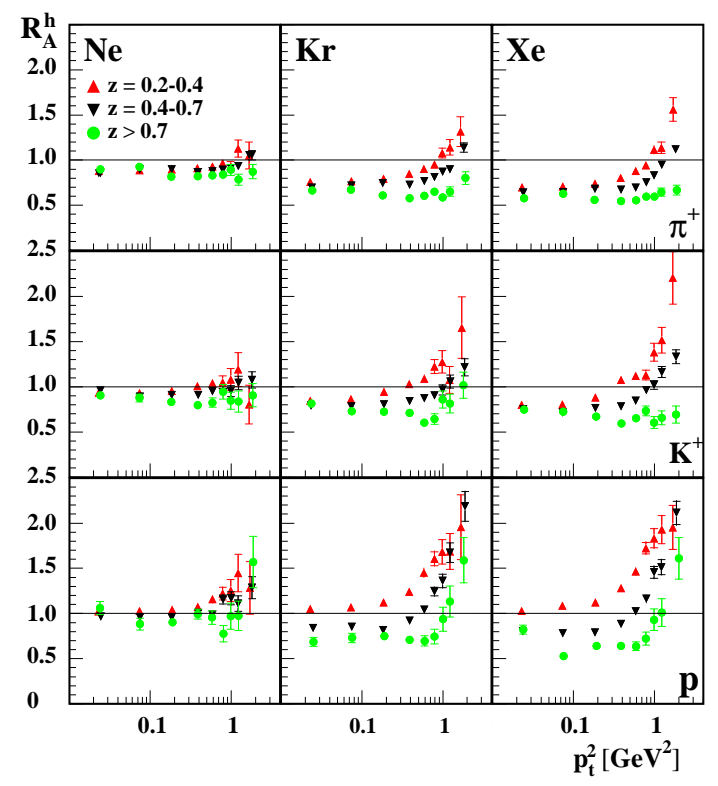

Figure 6: Dependence of $R_{A}^{h}$ on $p_{t}^{2}$ for three slices in $z$.

The $z$ dependence of $R_{A}^{h}$ for three slices in $p_{t}^{2}$ is presented in figure 7. The overall reduction of $R_{A}^{h}$ 
with $z$ was observed. At small values of $z$ a strong dependence of $R_{A}^{h}$ on $p_{t}^{2}$ was found while for high $z$ values there is almost no $p_{t}^{2}$ dependence for $\pi^{+}, \pi^{-}$and $K^{+}$.
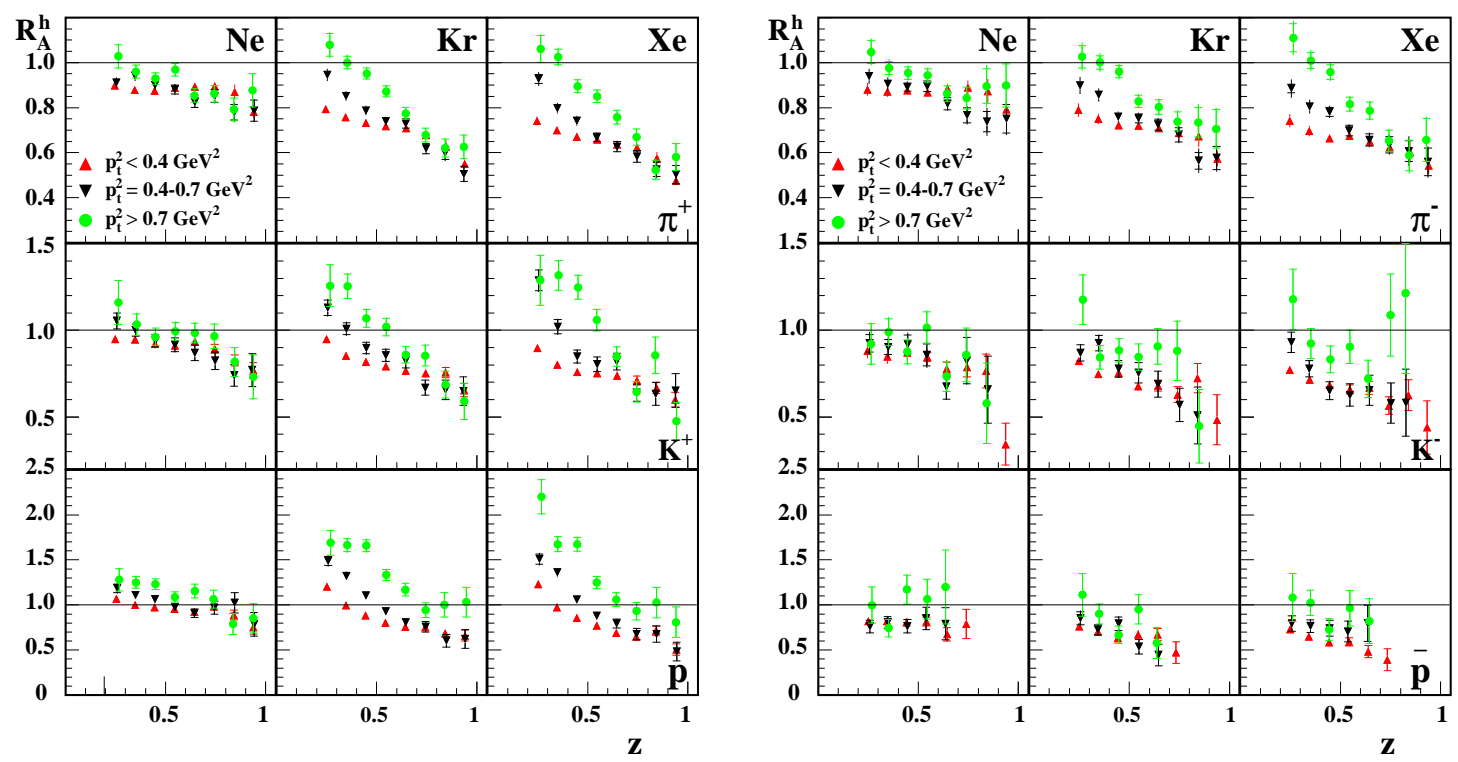

Figure 7: Dependence of $R_{A}^{h}$ on $z$ for three slices in $p_{t}^{2}$.

\section{Conclusions}

The first two dimensional kinematic dependencies for hadron multiplicity ratio $R_{A}^{h}$ have been presented for pions $\left(\pi^{+}, \pi^{-}\right), \operatorname{kaons}\left(K^{+}, K^{-}\right)$and $\operatorname{protons}(p, \bar{p})$ on neon, krypton and xenon targets relative to deuterium. For $\pi^{+}$and $\pi^{-}$the behaviour of $R_{A}^{h}$ was about the same within the experimental uncertainties. The dependence of $R_{A}^{K^{+}}$on $v$ for positevely charged kaons was found to be different from $R_{A}^{\pi^{+}}, R_{A}^{\pi^{-}}$and $R_{A}^{K^{-}}$at high values of $z$ which might be the result of final-state interactions. Proton data show a significant difference from the other hadrons which can be explained by a contribution of knock-out processes, in addition to the fragmentation process.

\section{References}

[1] L. Osborne et al., Phys. Rev. Lett. 40, 1624 (1978).

[2] J. Ashman et al. [EMC], Z. Phys. C 52, 1 (1991).

[3] A. Airapetian et al. [HERMES], Eur. Phys. J. C 20, 479 (2001).

[4] A. Airapetian et al. [HERMES], Phys. Lett. B 577, 37 (2003).

[5] A. Airapetian et al. [HERMES], Nucl. Phys. B 780, 1(2007).

[6] K. Akertaff et al., HERMES coll., Nucl. Instrum. Methods A417 239 (1998).

[7] N. Akopov et al. NIM A479 (2002) 511.

[8] A. Accardi et al., Riv. Nuovo Cim. 32, 439 (2010). 